\title{
A Bone-Breaking Case of Syncope Due to Glossopharyngeal Neuralgia
}

\author{
Christina Austin ${ }^{\mathrm{a}}$, Keerthana Karumbaiah ${ }^{\mathrm{a}}$, Bassam Omar ${ }^{\mathrm{a}, \mathrm{b}}$
}

\begin{abstract}
Glossopharyngeal neuralgia is an uncommon cause of intense facial pain, and is rarely associated with syncope. We present a patient with malignant syncope precipitated by glossopharyngeal neuralgia (GPN), who sustained lower extremity fractures. A 64-year-old Caucasian female with hypertension, diabetes mellitus type 2 and dyslipidemia, presented to the emergency department (ED) after a witnessed syncopal event. Over the preceding 9 months, she experienced multiple syncopal episodes triggered by neck pain, and was diagnosed with GPN by an ENT specialist. She reported a severe, stabbing pain in the right side of her neck prior to the event. Her vital signs and physical examination were unremarkable, except for bilateral leg tenderness. Laboratory data were within normal limits. Electrocardiogram revealed normal sinus rhythm, a rate of 72 beats per minute (BPM), and otherwise normal. Lower extremity X-rays revealed bilateral non-displaced fibular fractures. In the ED, she sustained another syncopal event, and bradycardia with transient heart rates as low as 22 BPM were documented. A temporary transvenous pacemaker was placed. She required pacing repeatedly for repeated bradycardic episodes preceded by right-sided neck pain. She was started on carbamazepine, without complete resolution of her symptoms on escalating doses. Due to recurrent bradycardia with frequent neck pains, requiring temporary pacing, an MRIcompatible permanent pacemaker was placed, to allow for further MRI recommended by neurology service. At 8-week follow-up, she had fully recovered from her neuralgic pain and fractures, without further syncope. This case emphasizes the potentially serious morbidity of GPN-induced syncope and importance of a prompt clinical diagnosis and treatment.
\end{abstract}

Keywords: Glossopharyngeal neuralgia; Syncope; Bradycardia; Pacemaker; Bone fracture

\footnotetext{
Manuscript accepted for publication July 24, 2014

${ }^{a}$ University of South Alabama, Mobile, AL 36617, USA

${ }^{\mathrm{b}}$ Corresponding Author: Bassam Omar, University of South Alabama, 2451 Fillingim Street, Mobile, AL 36617, USA.

Email: bomar@health.southalabama.edu
}

doi: http://dx.doi.org/10.14740/jmc1875w

\section{Introduction}

Glossopharyngeal neuralgia (GPN) is an uncommon cause of intense facial pain, and is rarely associated with syncope. GPN-associated syncope has been attributed to a combination of vasodepressor and cardioinhibitory mechanisms. Such syncopal episodes tend to be debilitating and may result in injury, necessitating aggressive neuropathic pain management and, in refractory cases, insertion of a permanent pacemaker. We present a patient who sustained lower extremity fractures due to malignant syncope precipitated by GPN, necessitating the insertion of a permanent pacemaker. We review the pertinent literature with regard to the incidence, mechanism, triggers, reported complications and potential therapeutic options of this rare entity.

\section{Case Report}

A 64-year-old Caucasian female with hypertension, diabetes mellitus type 2 and dyslipidemia, presented to the emergency department (ED) after a witnessed syncopal event. Over the preceding 9 months, she experienced multiple syncopal episodes triggered by neck pain, and was diagnosed with GPN by an ENT specialist. She reported a severe, stabbing pain in the right side of her neck prior to the event. Her vital signs and physical examination were unremarkable, except for bilateral leg tenderness. Laboratory data were within normal limits. Electrocardiogram revealed normal sinus rhythm, a rate of 72 beats per minute (BPM), and otherwise normal. Lower extremity X-rays revealed bilateral non-displaced fibular fractures (Fig. 1). In the ED, she sustained another syncopal event; bradycardia with heart rates as low as 22 BPM were documented, and ECG performed promptly revealed sinus bradycardia (Fig. 2). A temporary transvenous pacemaker was placed. She required pacing repeatedly for repeated bradycardic episodes preceded by right-sided neck pain. The episodes could be precipitated by opening her mouth, talking and application of pressure on the right side of neck. Due to the severity of the symptoms, the patient often communicated by writing on a note pad instead of talk- 


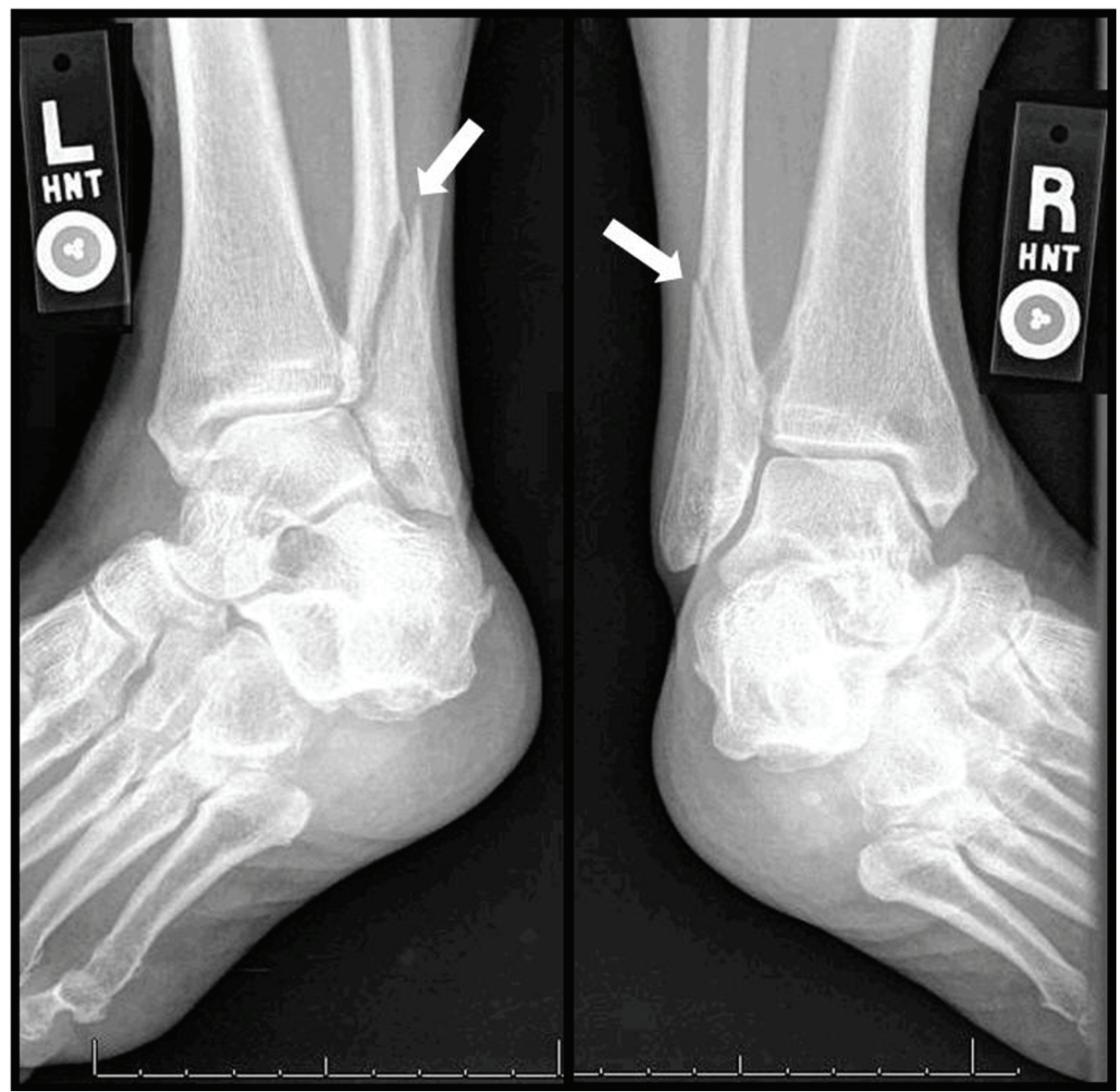

Figure 1. Bilateral foot X-rays reveal fibular fractures (white arrows).

ing, and also declined examination of oral cavity by physicians. She was started on carbamazepine, without complete resolution of her symptoms. Due to recurrent bradycardia with frequent neck pains, requiring temporary pacing, an MRI-compatible permanent pacemaker was placed, to allow for further MRI recommended by neurology service. At the time of her discharge from the hospital, she had a modest improvement in the severity of her neuralgic pain on an escalated dose of carbamazepine with reduction in frequency of episodes. At 8-week follow-up, she had fully recovered from her neuralgic pain and fractures, without further syncope.

\section{Discussion}

GPN causes paroxysmal attacks of brief stabbing pain, localized to the external ear canal, the base of the tongue, the tonsil or the area beneath the angle of the jaw, with triggers such as speaking, swallowing, eating, breathing, cold air and slight touch of the mouth and pharyngeal region [1]. Although it is mostly idiopathic without major pathology, and often caused by compression of glossopharyngeal nerve as it exits or enters the brainstem, secondary causes have been described including trauma, neoplasm, infection or vascular malformations [2]. GPN is rare in the general population, with an incidence rate of approximately 0.7 per 100,000 [3].

Syncope has been reported in four out of 216 GPN cases (approximately 2\%) of a Mayo case series [4]. The syncope appears to be hemodynamically mediated, with a drop in blood pressure and heart rate coinciding with the onset of pain, as demonstrated by Kim et al in a 53-year-old man admitted with recurrent syncope associated with pain from GPN [5]. One plausible mechanism explaining the association between bradycardia, hypotension and syncope, and GPN is the close connection between the vagus nerve and glossopharyngeal nerve with respect to visceral sensations, creating a vagoglossopharyngeal reflex arch [6]. Barbash et al [7] examined the mechanism of syncope in a patient with GPN. They reported that while atropine prevented asystole (without relieving pain), blood pressure continued to decrease due to inhibition of the sympathetic tone, with decrease in the serum norepinephrine levels during the attacks. Weinstein et al [8] also reported a case of GPN due to metastatic head and neck tumor with hypotension but without 


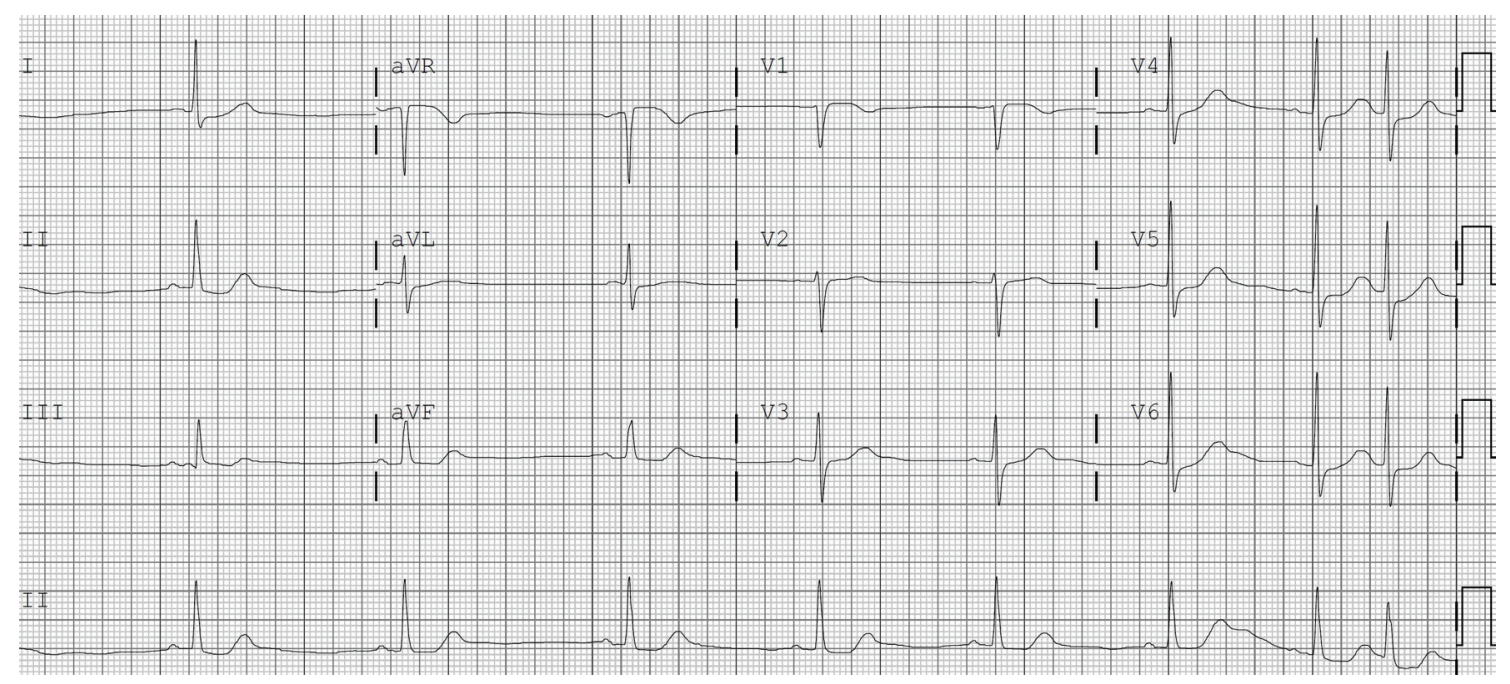

Figure 2. ECG following syncopal episode revealing significant sinus bradycardia.

bradycardia. Atropine resulted in sinus tachycardia but did not abolish the hypotension, suggesting selective stimulation of baroreceptor vasodepressor activity.

However, pain may not always be the trigger for the syncope seen in GPN. Korkes et al [9] reported a 71-yearold male with sporadic syncopal episodes, who complained only of ill-characterized spasms in the throat. A loop monitor revealed $4.4 \mathrm{~s}$ sinus arrest with presyncope while eating; his symptoms resolved after placement of a permanent pacemaker. Reddy et al [10] reported another case of syncope necessitating a permanent pacemaker insertion in a patient with GPN, but without pain in the distribution of the glossopharyngeal nerve.

The consequences of GPN-induced syncope vary. Our patient sustained bilateral fibular fractures due to an episode of GPN-induced malignant syncope. She also had stopped communicating verbally due to induction of pain while talking, and these symptoms were relieved with carbamazepine at 8-week follow-up. GPN patients may avoid eating, out of fear of chewing-induced pain and syncopal attacks, which can lead to weight loss. Odeh and Oliven [11] reported a 50 -year-old woman who lost $6 \mathrm{~kg}$ in 2 weeks because she refused to eat and drink, following 3 months of episodic pain in the left side of her throat precipitated by swallowing, which gradually progressed to loss of consciousness several times a day. Therapy with carbamezepine abolished the pain and syncope, and allowed restoration of her weight over 12 months. GPN-induced syncope may expose the patient to risk of automobile accidents, as in the report by Dunn et al [12] of a 60-year-old male with multiple episodes of syncope from GPN, refractory to medical therapy. While the patient was driving to the nearest ED, after having experienced six syncopal episodes in 1 day, he developed another episode and had just enough time to pull off to the side of the road before losing consciousness. Bodily injury can also be a complication of GPN-induced syncope, as in our patient. Elias et al [13] reported an 80-year-old white male with GPN who sustained head trauma and right maxillary sinus fracture following a syncopal episode triggered by lancinating pain in the pharynx. Seizures have also been reported in patients with GPN-induced syncope, likely due to the transient hemodynamic compromise and decrease in cerebrovascular perfusion [14-16].

Therapy for GPN-induced syncope varies among reports. In some reports $[17,18]$ treatment with carbamazepine abolished the pain and associated cardiovascular manifestations. However, in other cases, treatment with carbamazepine alone $[19,20]$, carbamazepine and gabapentin combination [21] or clonazepam [22] was not sufficient to alleviate the pain and syncopal episodes in GPN, necessitating the insertion of a permanent pacemaker to prevent syncope. Other successful treatments of refractory cases included section of the ninth (glossopharyngeal) nerve alone [23, 24], or combined with rhizotomy and microvascular decompression of the lower cranial nerves [25].

Our patient's syncope resulted in severe bodily injury causing lower extremity fractures. MRI of the head and neck was recommended by neurology service. However, since the presence of temporary myocardial pacing leads is considered a safety contraindication for MRI [26], it was deemed unsafe for her to undergo MRI testing due to her dependence on temporary pacing. Therefore, the permanent pacemaker brand inserted was REVO (Medtronic), which is compatible with 1.5-Tesla MRI scanners [27]. She later was discharged in good condition, and was scheduled to undergo an outpatient MRI for further evaluation.

\section{Conclusion}

In conclusion, GPN is a rare, but serious cause of cardiac 
syncope, which is associated with a well-defined presentation. It is paroxysmal, but episodes can be frequent enough to result in bodily injury. Treatment may address pharmacologic or surgical pain relief, versus addition of a pacemaker in refractory cases to prevent episodic asystole. Insertion of a novel MRI-safe pacemaker allows for safe future use of MRI for further workup when indicated.

\section{References}

1. Gaul C, Hastreiter P, Duncker A, Naraghi R. Diagnosis and neurosurgical treatment of glossopharyngeal neuralgia: clinical findings and 3-D visualization of neurovascular compression in 19 consecutive patients. J Headache Pain. 2011;12(5):527-534.

2. Singh PM, Kaur M, Trikha A. An uncommonly common: Glossopharyngeal neuralgia. Ann Indian Acad Neurol. 2013;16(1):1-8.

3. Katusic S, Williams DB, Beard CM, Bergstralh E, Kurland LT. Incidence and clinical features of glossopharyngeal neuralgia, Rochester, Minnesota, 1945-1984. Neuroepidemiology. 1991;10(5-6):266-275.

4. Rushton JG, Stevens JC, Miller RH. Glossopharyngeal (vagoglossopharyngeal) neuralgia: a study of 217 cases. Arch Neurol. 1981;38(4):201-205.

5. Park KJ, Choi NC, Kim SK, Kang H, Kwon OY, Lim BH. Teaching neuroimages: glossopharyngeal neuralgia with syncope: heart rate and blood pressure change. Neurology. 2011;77(14):e84.

6. Ferrante L, Artico M, Nardacci B, Fraioli B, Cosentino F, Fortuna A. Glossopharyngeal neuralgia with cardiac syncope. Neurosurgery. 1995;36(1):58-63; discussion 63.

7. Barbash GI, Keren G, Korczyn AD, Sharpless NS, Chayen M, Copperman Y, Laniado S. Mechanisms of syncope in glossopharyngeal neuralgia. Electroencephalogr Clin Neurophysiol. 1986;63(3):231-235.

8. Weinstein RE, Herec D, Friedman JH. Hypotension due to glossopharyngeal neuralgia. Arch Neurol. 1986;43(1):90-92.

9. Korkes H, de Oliveira EM, Brollo L, Hachul DT, Andrade JC, Peres MF, Schubsky V. Cardiac syncope induced by glossopharyngeal "neuralgia": a rare presentation. Arq Bras Cardiol. 2006;87(5):e189-191.

10. Reddy K, Hobson DE, Gomori A, Sutherland GR. Painless glossopharyngeal "neuralgia" with syncope: a case report and literature review. Neurosurgery. 1987;21(6):916-919.

11. Odeh M, Oliven A. Glossopharyngeal neuralgia associated with cardiac syncope and weight loss. Arch Otolaryngol Head Neck Surg. 1994;120(11):1283-1286.

12. Dunn Y, Oehler R, Small M, White G, Bruton A. Syncope from an Unusual Cause: Glossopharyngeal Neu- ralgia. Fed Pract. 2010;27(4):26-31.

13. Elias J, Kuniyoshi R, Carloni WV, Borges MR, Peixoto CA, Pimentel D. Glossopharyngeal neuralgia associated with cardiac syncope. Arq Bras Cardiol. 2002;78(5):510519.

14. Roulhac GE, Levy I. Glossopharyngeal neuralgia associated with cardiac arrest and convulsions. Arch Neurol Psychiatry. 1950;63(1):133-139.

15. Garretson HD, Elvidge AR. Glossopharyngeal neuralgia with asystole and seizures. Arch Neurol. 1963;8:26-31.

16. Kong Y, Heyman A, Entman ML, McIntosh HD. Glossopharyngeal Neuralgia Associated with Bradycardia, Syncope, and Seizures. Circulation. 1964;30:109-113.

17. Saviolo R, Fiasconaro G. Treatment of glossopharyngeal neuralgia by carbamazepine. Br Heart J. 1987;58(3):291292.

18. Ribeiro RT, Souza NA, Carvalho Dde S. Glossopharyngeal neuralgia with syncope as a sign of neck cancer recurrence. Arq Neuropsiquiatr. 2007;65(4B):1233-1236.

19. Johnston RT, Redding VJ. Glossopharyngeal neuralgia associated with cardiac syncope: long term treatment with permanent pacing and carbamazepine. Br Heart J. 1990;64(6):403-405.

20. Kim SH, Han KR, Kim do W, Lee JW, Park KB, Lee JY, Kim C. Severe pain attack associated with neurocardiogenic syncope induced by glossopharyngeal neuralgia: successful treatment with carbamazepine and a permanent pacemaker -a case report. Korean J Pain. 2010;23(3):215-218.

21. Kazemi B, Akbarzadeh F. Syncopal storm caused by glossopharyngeal neuralgia. Am J Emerg Med. 2012;30(9):2101 e2105-2107.

22. Hie CH, Arnold AER, Ruiter JH. Glossopharyngeal neuralgia with syncope: a case report. Neth Heart J. 2002;10(3):150-153.

23. Taylor PH, Gray K, Bicknell PG, Rees JR. Glossopharyngeal neuralgia with syncope. J Laryngol Otol. 1977;91(10):859-868.

24. St John JN. Glossopharyngeal neuralgia associated with syncope and seizures. Neurosurgery. 1982;10(3):380383.

25. Ozenci M, Karaoguz R, Conkbayir C, Altin T, Kanpolat Y. Glossopharyngeal neuralgia with cardiac syncope treated by glossopharyngeal rhizotomy and microvascular decompression. Europace. 2003;5(2):149-152.

26. Pfeil A, Drobnik S, Rzanny R, Aboud A, Bottcher J, Schmidt P, Ortmann C, et al. Compatibility of temporary pacemaker myocardial pacing leads with magnetic resonance imaging: an ex vivo tissue study. Int J Cardiovasc Imaging. 2012;28(2):317-326.

27. Bhandiwad AR, Cummings KW, Crowley M, Woodard PK. Cardiovascular magnetic resonance with an MR compatible pacemaker. J Cardiovasc Magn Reson. 2013;15:18. 